AIAA-2001-0235

\title{
THE LIVING WITH A STAR PROGRAM NASA'S ROLE IN ASSURING PERFORMANCE IN SPACE AND ATMOSPHERIC ENVIRONMENTS
}

\author{
Janet L. Barth, Member AIAA, NASA/Goddard, Greenbelt, MD \\ Kenneth LaBel, Member AIAA, NASA/Goddard, Greenbelt, MD \\ Dana Brewer, NASA/Headquarters, Washington, DC \\ George Withbroe, NASA/Headquarters, Washington, DC \\ Billy Kauffman, NASA/Marshall, Huntsville, AL
}

\begin{abstract}
NASA has initiated the Living with a Star (LWS) Program to develop the scientific understanding to address the aspects of the Connected Sun-Earth system that affect life and society. A goal of the program is to bridge the gap between science, engineering, and user application communities. This will enable future science, operational, and commercial objectives in space and atmospheric environments by improving engineering approaches to the accommodation and/or mitigation of the effects of solar variability on technological systems. A pre-formulation study determined the optimum combination of science missions, modeling, and technology infusion elements to accomplish this goal. The results of the study are described below.
\end{abstract}

\section{INTRODUCTION}

The Sun, the astronomical object most significant to humanity, affects the entire geospace region. Because of the consequences to the Earth of the Sun's dynamic behavior and the rapidly expanding utilization of this region for human activities, a thorough understanding of the Sun's effects is becoming increasingly essential. The Sun radiates both as electromagnetic energy and as fast-moving electrically charged particles. The electromagnetic radiation across a broad spectrum of wavelengths originates from the photosphere, the Sun's surface. This energy proceeds unimpeded directly from the Sun to the Earth's atmosphere, with a majority reaching the surface of the Earth.

The streaming of electrically-charged particles away from the Sun results from the energizing of gases in the solar corona. This continuous, but highly variable stream, called the solar wind, has speeds of up to $1000 \mathrm{~km}$ per second. Its variability is closely connected to events on the Sun and its corona. In contrast to electromagnetic radiation, the transit of energy in this form through space is very complex, especially as it interacts with magnetospheres and atmospheres to produce a wide variety of phenomena and consequences. The Living with a Star (LWS) program will permit comprehensive study of the cause-and- effect relationships between events at the Sun and their effects in geospace that influence life on Earth and humanity's technological systems. ${ }^{1}$

The Connected Sun-Earth system has far reaching consequences for life and society. The specific areas of concern that the LWS Program will address can be summarized into three categories:

$$
\begin{array}{ll}
\text { - Human Radiation Exposure } \\
\text { - Space Station } \\
\text { - Space Exploration } \\
\text { - High Altitude Flight } \\
\text { - Space Utilization \& Colonization } \\
\text { Impacts on Life and Society } \\
\text { - Global Climate Change } \\
\text { - Surface Warming } \\
\text { - Ozone Depletion and Recovery } \\
\text { Impacts on Technology } \\
\text { - Space Systems } \\
\text { - Aircraft Systems } \\
\text { - Communications and Navigation } \\
\text { - Ground Systems }
\end{array}
$$

The goal of the LWS program is to develop scientific understanding of the Connected Sun-Earth system under a program architecture that will increase the flow of scientific understanding to enabling science disciplines. Using the data and information generated by the program, enabling science will have significantly improved capability to address the mitigation of effects induced by solar variability. Fig. 1 illustrates the application areas that will benefit from the results of the LWS Program. The impact of the LWS Program is broad and far reaching. This purpose of this paper is to describe the role of the program in improving capability and reliability in space and atmospheric environments.

\section{ASSURANCE OF SYSTEM PERFORMANCE}

The complex environment of Sun-Earth space consists of time varying ultraviolet, $x$-ray, plasma, and high energy particle environments. The particle environment is composed of fluxes of electrons with energies up to $10 \mathrm{~s}$ of $\mathrm{MeV}$ and protons and heavier ions with energies up to $10,000 \mathrm{~s}$ of $\mathrm{MeV}$. Variations depend 
on location in space and on the year in the solar cycle, both somewhat predictable. However, large variations that depend on events on the Sun are not predictable with reasonable certainty and are known only statistically based on past history.

The natural space environment and its solar induced variability pose a difficult challenge for designers of technological systems. Space and atmospheric environments interact with aircraft and spacecraft components to induce effects in systems. The effects include degradation of materials, thermal changes, contamination, excitation, spacecraft glow, charging, communication and navigation errors and dropouts, and radiation damage and induced background interference. The accommodation of environmentally induced effects is accomplished in design and operational phases of system development.

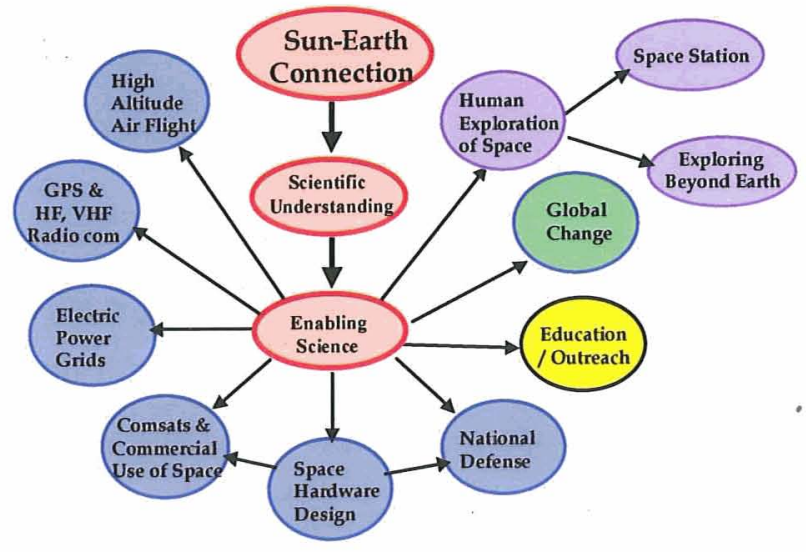

Fig. 1: The flow of scientific understanding to application areas

The prediction of the performance of technologies in space is obtained from models, which simulate the interaction of the space environment with technological components. The inputs to the prediction models include estimates of the space environments and the results of ground test protocols performed on the specific components. Design margins must be included in the predictions to account for inaccuracies in the prediction methods. Drivers for the size of the design margins are the fidelity of the simulation method to the actual environment and the accuracy of the two inputs, the environment and the ground test protocol.

Large uncertainties in space and atmospheric environments and effects models preclude cost effective use of environmentally sensitive technologies in the space environment. To successfully infuse a new technology into a system, accurate environment models, ground test protocols, and interaction models are required, and the models must be validated with inflight experiments. ${ }^{2}$
To enable system performance assurance, the LWS program will address the need to develop and validate more accurate prediction models and engineering tools. The LWS program plan will be described below. However, we will first discuss developments in spacecraft design practices that have been drivers for including spacecraft technology issues in the program.

\section{DEVELOPMENTS IN SPACECRAFT DESIGN PRACTICES}

The approach to the design of spacecraft has changed completely since the development of most of the currently available prediction methods and their space environment and ground test inputs. Design practices that have an impact on understanding technology performance are described below.

\section{DEMISE OF THE ENVIRONMENT HARDENED MARKET}

In the 1970 s the view was widely held that designing systems that are immune to environment effects would become a "non-problem" with the development of inherently hardened components. For example, space programs relied on the development of radiation hardened components for military applications. However, the investment in rad-hard military programs has dropped significantly, and component manufactures have turned their attention to the commercial markets. In fact, the number of rad-hard foundries has decreased from eighteen to two or three. The result is that there are few radiation-hardened components available for spacecraft designs.

\section{COMMERCIAL DEMAND FOR ELECTRONICS}

The demand for electronics capacity in commercial markets has reduced the space market share from $40 \%$ to $0.4 \%$, and the growth potential for the space market is small. Therefore, manufactures are not driven to produce or qualify components specifically for use in the space environment greatly reducing the components available for applications in space, atmospheric, or nuclear environments.

\section{SHORTER MISSION DEVELOPMENT TIMES}

The length of time to get missions into orbit has decreased over the past decade. Development times as short as three years from concept to operations are not unusual. Custom components with controlled processes take too long to develop for these missions. This forces the reliance of parts procurements on commercial-offthe-shelf (COTS) components which are not space qualified. To be used in the space and atmospheric environments, COTS must be characterized using ground-test protocols. Unfortunately, small processing 
changes are frequently made to increase yield, which can radically change the component's response to the environment. Also, little tracking control on product lines implies there may be large variations in the response to space environments within a manufacturing lot.

\section{SMALLER, LIGHTER SPACECRAFT}

Another development in spacecraft technology that influences new modeling efforts is the advent of smaller, lighter, low power spacecraft. Essentially, we are dealing with a problem of designing satellites with greatly reduced built-in shielding while, at the same time, using parts that are more sensitive to space environments. This combination implies that there is less tolerance for large design margins than in the past.

\section{MORE DEMANDING MISSION REQUIREMENTS}

Missions are required to deliver more capability making requirements more demanding. Desired mission features include imaging, real-time operations, onboard processing, smart systems and autonomous operations, etc. Systems are more complex, need higher speed, and require large downlink budgets. Generally, these requirements cannot be met with the available space qualified technologies so designers rely on emerging technologies with performance characteristics that are difficult to characterize on the ground and which may be intolerant of space and atmospheric environments.

\section{DESIRE TO OPERATE IN MORE SEVERE ENVIRONMENTS}

A strategy to reduce risk is to avoid the intense part of the radiation belts by operating in low altitude regions where the exposure occurs intermittently due to passes through the South Atlantic Anomaly or above the intense part of the belts at geostationary positions which is well past the peak of the proton and electron trapping regions. Both science and commercial satellites would like to operate in higher radiation regions. Communication satellites can reduce the number of spacecraft in their constellations by increasing the altitude of the missions. For science missions, flying in low altitude regions reduces the time available for science observations but cost often limits the opportunity to launch beyond low altitudes. Due to the higher radiation in middle earth orbits (MEOs), large design margins imposed by inaccurate models cannot be tolerated. Also, the radiation environment is more dynamic at higher altitudes where storms can cause sudden increases in the particle levels.

Longer mission durations are also desirable. Longer durations increase exposure to degradation environments and increase the probability of catastrophic events. Increasing mission durations can drive even low earth orbiters to environment levels that are too high for use of environmentally soft technologies.

\section{CONSEQUENCES IN CHANGE IN DESIGN PRACTICES}

The demise of the space qualified component market, the commercial demand for electronics, shorter development times, use of smaller and lighter spacecraft, more demanding mission requirements, and the desire to operate in all regions of space all contribute to the use of more sensitive commercial off the shelf (COTS) components and emerging technologies in more severe space environments. In other words, component choices are driven by capability and availability and not by qualification for use in space and atmospheric environments. For the most part, these technologies are not space-qualified by manufacturers, and they tend to be increasingly sensitive to the environment. The trend toward using more sensitive technologies in technological systems implies that we can no longer completely avoid risk but must rely on managing risk. To accomplish that task, better understanding of the space and atmospheric environments and the interactions with components is required.

Risk avoidance and management for space systems are traditionally achieved through system design and component screening. However, increasingly, designing capable spacecraft that are $100 \%$ free from environment-induced effects cannot be accomplished within reasonable costs. As a result, we are forced to develop systems that must accommodate space environment effects during on-orbit operations.

\section{THE LIVING WITH A STAR PROGRAM}

The LWS program seeks to address the issues of inaccurate prediction models and the need to reduce and manage risk associated with the effects of solar variability on technology. The program is a crosscutting initiative whose goals and objectives have the following links to each of the four NASA Strategic Enterprises:

- Space Science: LWS quantifies the physics, dynamics, and behavior of the Sun-Earth system over the 11-year solar cycle.

- Earth Science: LWS improves understanding of the effects of solar variability and disturbances on terrestrial climate change.

- Human Exploration and Development: LWS provides data and scientific understanding 
required for advanced warning of energetic particle events that affect the safety of humans.

- Aeronautics and Space Transportation: LWS provides detailed characterization of radiation environments useful in the design of more reliable electronic components for air and space transportation systems.

The LWS program is designed to address environment accommodation issues for future spacecraft. The program architecture, illustrated in Fig.2, consists of the following elements:

- Science Missions to collect data,

- Theory and Modeling to define the external environment,

- Space Environment Testbeds (SET) to define the environment interaction with spacecraft components, and

- Evolution of established and expanded partnerships.

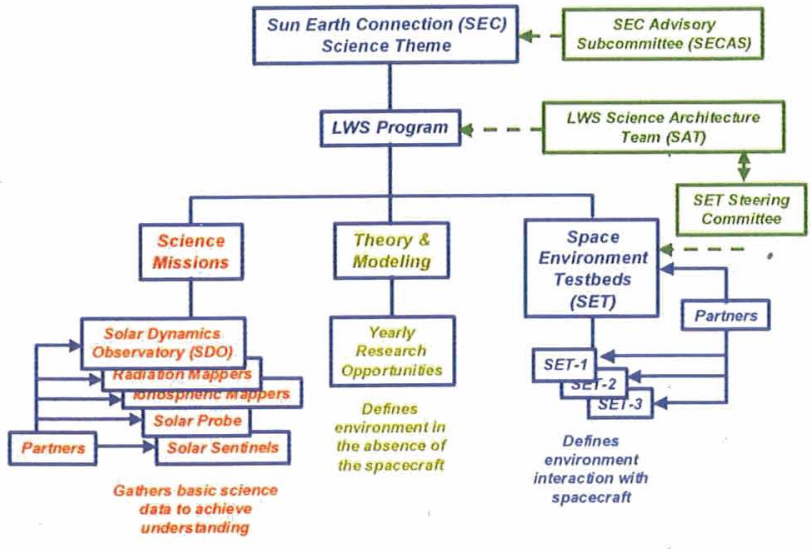

Fig. 2: This figure illustrates the program architecture of the LWS program.

\section{SCIENCE MISSIONS}

The purpose of the Science Missions is to collect data for scientific understanding of solar variance and space weather and for use by modeling programs. There are two groups of mission spacecraft in the LWS science missions: (a) solar dynamics elements (Solar Dynamics Observer/Solar Sentinels) that observe the Sun and track disturbances originating there and (b) geospace dynamics elements (Radiation Belt Mappers/ Ionospheric Mappers) consisting of constellations of small satellites located in key regions around the Earth to measure downstream effects.

Solar Dynamics Observatory will observe the Sun's dynamics and help us understand the nature and source of variations, from the stellar core to the turbulent solar atmosphere. Sentinels will provide a global view of the heliosphere and describe the transition and evolution of eruptions and flares from the Sun to Earth. Radiation Belt Mappers will help us to understand the origin and dynamics of the radiation belts and determine the evolution of penetrating radiation during magnetic storms. Ionospheric Mappers is a global network of satellites that will gather knowledge of how the ionosphere behaves as a system, linking solar energy with the Earth's atmosphere.

Fig. 3 shows one concept for the science mission network. The goal is to have the entire system in place for a portion of the next solar maximum.

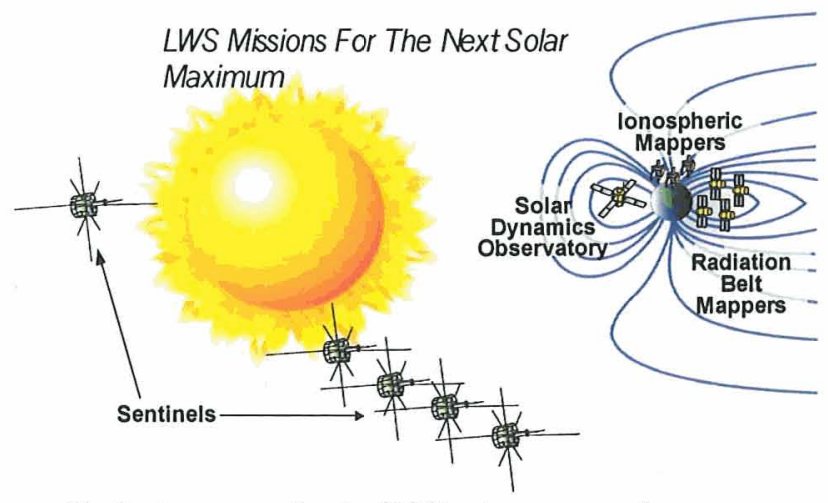

Fig.3: A concept for the LWS science network

\section{THEORY AND MODELING MISSION}

The objective of the Theory and Modeling element is to perform research to refine the understanding of space weather and the role of solar variability in terrestrial climate change. The goals are to improve understanding of space weather and solar variability, improve understanding of solar variability and its effect on long term climate change, and to perform research and development to enable improved environment specification models and predictive capability. The Theory and Modeling element will enable technological application areas by defining the space and atmospheric environment in the absence of the aircraft or spacecraft.

Environment models for enabling science disciplines need to be designed to address four areas:

- System Design

- Mission Planning

- Risk Management

- On-orbit Operations

Each of these factors imposes different requirements on space environments and effects models. System design requires accurate specification models so low design margins can be applied to performance predictions. 
As we saw in the section above, factors that drive design practices imply that designs that $100 \%$ free from environmentally induced effects are increasingly unrealistic. As a result, specification models alone do not describe the environment to the degree required. Mission planning requires models that give the time distribution of environment variability so observation times, system degradation factors, and data corruption can be assessed. Also, program managers must make risk management decisions based on probability models. Finally, on-orbit operations will rely on space weather models that provide forecasting for protection of systems and reconfiguration operations and nowcasting for anomaly analysis and situational awareness.

\section{LWS SPACE ENVIRONMENT TESTBEDS}

The Space Environment Testbeds (SET) concept is being defined to complete the transition from science to applications with the objective of improving the engineering approach to accommodate and/or mitigate the effects of solar variability on spacecraft design and operations. The SET has three goals. The first is to enhance the technical and scientific capability of satellite systems by enabling easy, low cost, and fast access to space for technology validation in the relevant environment. The second goal is to function as a pathfinder for future spacecraft deployment in space environments for commercial, government, and science interests by improving environment definitions and effects models and guidelines. The goals will be discussed in more detail in the next sections. The last goal is to infuse the improved predictive capability and validation results in space and atmospheric environments to government and industry users for space weather prediction, spacecraft design and operations, and terrestrial/ aircraft operations. These goals will be discussed in more detail in the next sections.

A concept study for space environment testbeds that would achieve these goals and provide data to improve the environment models was developed under the Orbiting Technology Testbed Initiative (OTTI) before the LWS program was begun. ${ }^{3}$ Because the LWS science missions and the Theory and Modeling Project now have the responsibility to define the environment, the trade space for the testbeds has changed significantly. As a result, new concept studies are now underway to refine a testbed concept for the LWS/SET program.

Goal 1: Technology Validation in the Relevant Environment

The capability to use COTS and emerging technologies in harsher the space environments does not exist at the present time. Enabling the capability requires:

- Defining the environment and its effects on spacecraft performance;

- Defining a sequence of testbeds and orbits that expose new technologies to the appropriate environment in a stepwise approach;

- Determining issues associated with on-orbit validations of new technologies;

- Defining the launch options and mission operations architectures; and,

- Identifying potential customers.

The OTTI concept study identified a low inclination, highly elliptical orbits as the optimal environment for the OTTI mission testbed. Because the testbeds are now part of the LWS Program, the requirement of the testbeds to characterize the environment is now an objective of the LWS Science missions. As a result, the orbit options are much broader for the testbeds and launch opportunities that provide environment exposure specific to SET payloads can be considered. For example, polar low earth orbits are good for single event effects investigations, geostationary and geostationary transfer orbits are good for ionizing dose and spacecraft charging experiments, and MEOs are good for understanding displacement damage.

\section{Environments Requirements}

An OTTI environments requirements trade study was performed to define orbits that provide the desired level of radiation effects on electronic systems and to define the level of measurements of the environment needed to interpret and model radiation effects and to improve performance prediction methods. These requirements are directly applicable to the SET. Components of the environment that are desirable exposures for SET experiments are:

- Plasma to characterize surface charging;

- Trapped protons for SEEs and degradation from total ionizing dose and displacement damage;

- Trapped electrons for internal charging, dose, and degradation; and,

- Transient protons and heavier ions from galactic cosmic rays (GCRs) and solar events for SEEs, dose, and degradation.

The ideal complement of environment measurement devices to provide adequate correlation of environment effects for the SET experiments includes:

- Electron, proton, and cosmic ray detection at to be determined energy levels;

- Dosimetry distributed throughout the spacecraft; 
- High and low LET spectra;

- Electrostatic discharge;

- Micrometeoroid measurements.

\section{Payload Requirements}

Rapid technology infusion into new missions requires a combination of space qualification and ground testing. Comparisons of the emerging and potentially competing technologies and new system architectures also require space qualification and in-flight comparisons. Examples of competing technologies are technologies for high-speed data transfer, infrared, ultraviolet, and visible sensors, solar cells, and microelectromechanical (MEMS) devices. System architectures include reconfigurable computing (RC), and on-board autonomy such as algorithmic logic, neural nets, and fuzzy logic. Candidate technologies are described in more detail in Reference 2.

A testbed concept (Fig. 4) contains three types of components based upon customer requirements: environments measuring devices, box experiments, and board experiments. Requirements for environment measurements were described in the previous section. Box experiments are independent, self-contained experiments in separate packages that are provided by the customer. They interface directly to the testbed or to the host spacecraft for power and data. In the OTTI study, the weight and power for each box experiment was scoped at several to 10 kilograms and 20 to 25 Watts. These parameters will be reconsidered in the LWS/SET concept analyses.

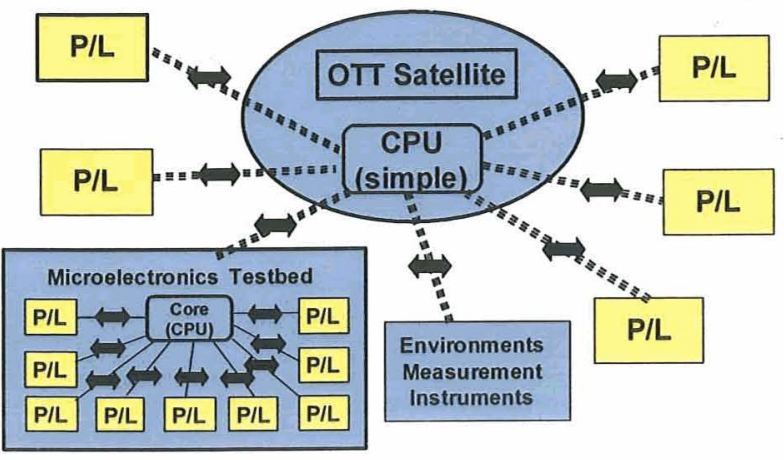

Fig. 4: A testbed concept.

Board experiments are modular card experiments that connect to a central or motherboard through a simple and common interface for analysis, storage and transfer of data and power conditioning. The customer provides them. Each board is typically five inches square, weighs $0.5 \mathrm{~kg}$, uses 15 to 20 Watts of peak power, and contains 10 to 20 devices. Orbit average power for each board experiment is about 3 Watts, and the smallest LWS concept contains 50 microelectronic, photonic, and subsystem board experiments.

\section{Goal 2: Improvement in Environment and Effects Models and Guidelines}

Uncertainties in spacecraft performance are derived from sources that can be reduced thereby reducing risk and cost and uncertainties that cannot be reduced. They are shown in Fig. 5. Natural variations in the space environment such as variations in solar activity cannot be reduced and must be accommodated or mitigated in the spacecraft design and/or operations. Uncertainties due to lack of accurate information about the definition of the environment or the performance of the spacecraft hardware in the environment can be reduced by:

- Obtaining more accurate environment definitions;

- Developing design and operations models and guidelines that better characterize the environment;

- Developing better definitions of the performance of the hardware in the environment; and,

- Developing better test and analysis capabilities to verify the hardware's performance in the spacecraft design and operations.

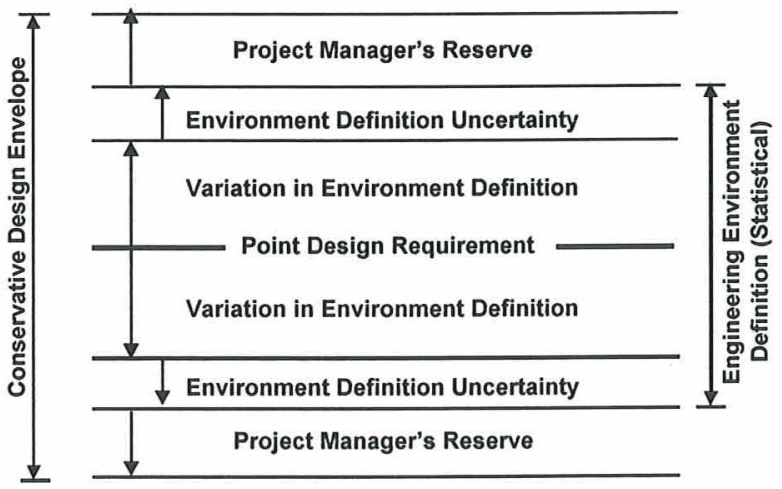

Fig. 5: State-of-practice for spacecraft design requirements and operations planning margins.

The LWS program seeks to reduce all four sources of uncertainty due to lack of accurate information and thus retire risk for using emerging technology. Reference 2 gives examples of areas where SET investigations can contribute to reductions in uncertainties in performance predictions. These reductions will permit reductions in the margin for uncertainty in the environment definition and in the project manager's reserve, thus reducing cost. In the Theory and Modeling element, it will use existing science data and data from the LWS Science missions that define the environments to improve the 
engineering environments definitions, particularly for plasma and ionizing radiation.

The performance of the hardware in the environment will be better defined by combining hardware testing in the space environment and correlative testing on the ground. The goal of the correlative testing is to reduce spacecraft risk and verification costs by developing a better ground test capability instead of always relying on the more costly testing in space. Models to characterize the hardware's performance in space and atmospheric environments will also be developed.

\section{System and Subsystem Testing in the Space Environment}

Uncertainties in predicting system and subsystem performance in the space environment can be reduced by flight validating the subsystems and systems in space as well as reducing the uncertainties in the predictive models for the space and atmospheric environment and its effects. Reductions in uncertainties will facilitate the routine use of MEO in the future.

\section{Infusion of Results to Spacecraft Design and}

\section{Operations}

The last goal, to infuse the results to spacecraft designers and operators, will be accomplished by sharing the results of the partner experiments with all participants and disseminating the results using publications. The improvement in the capability to predict spacecraft performance with reduced uncertainty margins will provide better, less costly, and more capable spacecraft than at the present time.

\section{THE SET IMPLEMENTATION PLAN}

The first task of the SET implementation plan is to establish a Steering Committee to represent the interests of NASA and the LWS/SET partners. The SET is a highly leveraged effort that requires strong partnerships to meet its goals. Therefore, NASA is seeking SET partners for both experiments and for the development of the testbeds. A function of the Steering Committee is to achieve agreement of the SET partners the on objectives and requirements of the SET. Three levels of partnership are envisioned. The first is LWS/SET program partners who participate in all SET aspects. These partners contribute to the success of the SET by taking part in the agreement process on objectives and requirements and participating in all program aspects. The second are testbed partners who contribute to the success of the SET. Testbed partners retain separate requirements and objectives and obtain allocation of spacecraft resources to achieve objectives. Finally, payload/experiment partners offer payloads in exchange for on-orbit operation, launch, and data return. A "Payload" includes ground test data if appropriate, on- orbit data after reduction, \& funding for integration and on-orbit operations. SET will have large flexibility in payload partnership guidelines and variations. Variations in definitions of "payloads" are negotiable and "funding" can include in kind exchanges. Partnership agreements can include Cooperative Agreements, fee for service, Memoranda of Agreements, and Space Act Agreements.

The SET is a multi-project plan with access to space offered every two years and mission durations of two years. Each project will contain several stand-alone experiments and correlative environments measurements. The short mission duration and launch cadence is planned in order to keep pace with technology changes by providing rapid access to space. Other in-flight experiment opportunities will also be investigated. If opportunities for rides occur in between projects, the plan is to have a reserve of investigation boards and carrier systems ready to take advantage of it. Bi-yearly SET workshops are planned to define requirements for requirements definition and partnering and presentations of results.

The experiments and environment detectors will be solicited by NASA Research Announcements (NRAs). The first solicitation is planned for early February, and the first testbed is expected to be ready for access to space in the late FY 2003/early FY 2004 time frame. The solicitation of proposals for experiments and environment detectors for follow-on testbed projects will occur in follow-on NRAs. The priorities for selection will be based on the quality of the research, the level of performance improvement and/or risk reduction offered by the results of the experiment, and the partner interest in the experiment. Support for the experiment builds for technologies of interest to NASA/Industry is planned.

The design for the experiment platform is a modular carrier that will be the interface between the SET or host spacecraft and the SET experiments. This flexible carrier system will be designed to fly on multiple platforms, which will provide interfaces to the host ride. Investigations will be boards supplied with technology being studied and there will be several different investigations in one carrier. A concept of the carrier system is shown in Fig. 6.

The experiments can be part of a microelectronics testbed or "standalone". The carrier will also hold the detectors required for correlative environments measurements. The environment detectors will be selected based on the payload. A carrier concept study is nearing completion at NASA and a trade study exercise will be performed in the second quarter of FY01. NASA's New Millenium Program is also performing a carrier study that the SET will take into consideration. 


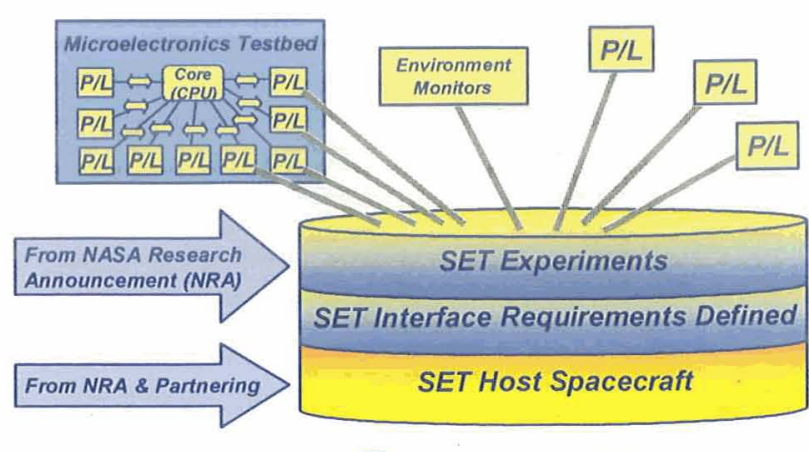

Fig 6: The interface concept for the SET

Appropriate candidates for the SET fall into two categories: SET space flight candidates and SET data analysis candidates. Space flight candidates must meet the following criteria:

- The technology must require space flight for performance or validation,

- The technology must applicable to more than one mission or to a LWS mission, and

- The performance of the technology must change due to the effects of solar variability and changing the spacecraft design cannot minimize the performance change.

The criterion for data analysis candidates is that the data must describe the performance variations in space in the presence of a spacecraft that changes due to solar variability.

The SET provides for post-flight data analysis and engineering tool, database, and guidelines development through the NRA process. It does not provide for technology development, ground testing for the experiments, or ground test protocol development. These functions must be leveraged from other programs, such as, NASA's Electronics Radiation Characterization (ERC) Project and DoD technology development programs.

\section{$\underline{\text { SUMMARY }}$}

NASA's Living with a Star program is a multidiscipline science research program that will take an integrated approach to understanding solar variability and its effects. An important area of research for LWS is to expand our understanding of the space and atmospheric environment and its effects on technological systems. This is largely driven by the rapid changes in technology and commercial demand for technology, which has far outpaced the research in enabling science disciplines.

We have shown the need for the four LWS program elements (the science missions, theory and modeling, space environment testbeds, and program partnering) to accomplish the goals of the program. The Space Environment Tesbeds will be designed to address issues related to rapid technological changes. The goal is to enable to use of COTS and emerging technologies to enhance system performance and decrease risk with reduced cost.

\section{REFERENCES}

${ }^{1}$ Living with a Star WEB Site http://sec.gsfc.nasa.gov/ lws.htm

${ }^{2}$ LaBel, K. A., J. L. Barth, D. Brewer, R. Howard, B. Kauffman, G. Giffin, C. Marshall, and P. Marshall, "The Effects of Solar Variability on Technology: Objectives of NASA's Space Environment Testbed," to be presented at IEEE Aerospace Conference, April 2001.

${ }^{3}$ Brewer, D. A., K. A. LaBel, J. C. Ritter, J. L. Barth, K. S. Clifton, S. D. Pearson, J. Peden, A. Campbell, and R. Liang, "The Orbiting Technology Testbed (OTTI) Space Experiments," RADCES Conference Proceeding, Fontevraud, France, September 13-17, 1999. 